\title{
Modulation of Angiotensin II-induced Vasoconstriction by Endothelium-derived Relaxing Factor in the Isolated Microperfused Rabbit Afferent Arteriole
}

Sadayoshi Ito, Cynthia S. Johnson, and Oscar A. Carretero

Hypertension and Vascular Research Division, Heart and Vascular Institute and Division of Biostatistics

and Research Epidemiology, Henry Ford Hospital, Detroit, Michigan 48202

\begin{abstract}
Although endothelium-derived relaxing factor (EDRF) has been studied extensively in large vessels, little is known about its role in the preglomerular afferent arteriole (Af-Art). We tested the hypothesis that EDRF, which is produced locally in the Af-Art, modulates arteriolar responses to angiotensin II (AII). A single rabbit Af-Art with its glomerulus intact was microperfused in vitro at $60 \mathrm{mmHg}$. When $0.1 \mu \mathrm{M}$ AII was first applied, luminal diameter decreased by $49 \pm 7.0 \%(n=9 ; P$ $<0.0001$ ); however, constriction waned, with the decrease becoming $15 \pm 3.5 \%$ at $1 \mathrm{~min}$. After washing the Af-Art, repeated AII caused less constriction $(13 \pm 4.0 \% ; P<0.0002$ vs. first application), showing tachyphylaxis. Pretreatment with $N^{\text {w }}$-nitro-L-arginine ( $N$-Arg), which inhibits synthesis of nitric oxide (an EDRF), decreased basal diameter by $18 \pm 3.0 \%(n=14 ; P$ $<0.0001)$. $N$-Arg also augmented AII-induced constriction $(86 \pm 6.8 \% ; P<0.02$ vs. nontreated Af-Art) and rendered it persistent $(82 \pm 6.9 \%$ at $1 \mathrm{~min})$. Even after pretreatment with $\boldsymbol{N}$-Arg, repeated AII caused a weaker response, which was restored by washing with kidney homogenate rich in angiotensinase. In conclusion, this study provides evidence that local production of EDRF is an important determinant of the tone of the Af-Art. Our results suggest that the transient nature of AII-induced constriction of the Af-Art may be due to production of EDRF, while tachyphylaxis may be the result of long lasting receptor occupancy. (J. Clin. Invest. 1991. 87:1656-1663.) Key words: Resistance vessel $\bullet N^{\text {" }}$-nitro-L-arginine $\bullet$ tachyphylaxis $\bullet$ prostaglandin $\bullet$ angiotensinase
\end{abstract}

\section{Introduction}

In 1980, Furchgott and Zawadzki (1) described the obligatory role of the endothelium in acetylcholine-induced relaxation of the arterial smooth muscle, suggesting the existence of endothelium-derived relaxing factor (EDRF). ${ }^{1}$ Since then, EDRF has been studied extensively in large vessels, and it has been shown that inhibition of EDRF or removal of the endothelium aug-

Address correspondence and reprint requests to Dr. Sadayoshi Ito, Hypertension and Vascular Research Division, Henry Ford Hospital, 2799 West Grand Boulevard, Detroit, MI 48202.

Received for publication 19 September 1990 and in revised form 7 December 1990

1. Abbreviations used in this paper: AII, angiotensin II; EDRF, endothelium-derived relaxing factor; LMMA, $N^{\mathrm{w}}$-monomethyl-L-arginine; $N$ $\mathrm{Arg}, N^{\mathrm{w}}$-nitro-L-arginine.

J. Clin. Invest.

(c) The American Society for Clinical Investigation, Inc.

$0021-9738 / 91 / 05 / 1656 / 08 \$ 2.00$

Volume 87, May 1991, 1656-1663 ments the action of various vasoconstrictors (2-4). However, to our knowledge, the role of EDRF in the control of preglomerular afferent arteriolar resistance has not been studied directly. Since the afferent arteriole is not only a small resistance vessel ( $\sim 20 \mu \mathrm{m}$ in diameter), but also a major component which regulates kidney function, it would be important to understand the action of EDRF in this vessel.

We have recently established an in vitro preparation consisting of a microperfused rabbit afferent arteriole with its glomerulus intact (Fig. 1). We found that in this preparation, angiotensin II (AII), a potent constrictor of the afferent arteriole (5-8), caused only a transient decrease in the luminal diameter, which diminished within 1 min despite the presence of AII (9). Furthermore, repeated applications of AII caused much weaker (though still transient) constriction compared with the first application (tachyphylaxis).

AII is well known to cause tachyphylaxis in many in vitro preparations (10-13); however, the underlying mechanism is not well understood. It has been shown that a plasma fraction rich in angiotensinase reverses AII tachyphylaxis (10). It is thought that this reversal is due to degradation of receptorbound AII, which in turn causes conformational changes of the receptor-AII complex and thereby facilitates dissociation. Thus it has been suggested that long lasting receptor occupancy may be responsible for AII tachyphylaxis. Other studies suggest that prostaglandin biosynthesis may be involved $(11,12)$.

In this study, we tested the hypothesis that the transient nature of the AII-induced constriction in our preparation is due to production of EDRF, while the tachyphylaxis is due to long lasting receptor occupancy by AII. For this purpose, we studied whether inhibition of either EDRF or prostaglandin biosynthesis and/or treating arterioles with kidney homogenate (which is rich in angiotensinase) alters the response to AII.

\section{Methods}

\section{Isolation and microperfusion of an afferent arteriole}

We used a method similar to that described in our previous papers to isolate the afferent arterioles (14-16). Briefly, young male New Zealand white rabbits $(1.0-1.2 \mathrm{~kg}$ ), fed standard rabbit chow (Ralston $\mathrm{Pu}$ rina Co., St. Louis, MO) and tap water ad libitum, were anesthetized with intravenous sodium pentobarbital $(40 \mathrm{mg} / \mathrm{kg})$ and given an intravenous injection of heparin $(500 \mathrm{U})$. The aorta was catheterized below the renal arteries and clamped with a hemostat above the kidneys. The kidneys were perfused with cold medium 199 (Gibco Laboratories, Grand Island, NY) containing 5\% BSA (Sigma Chemical Co., St. Louis, MO), then removed and sliced along the corticomedullary axis. Slices were placed in ice-cold medium 199 containing 5\% BSA (medium 199-5\%BSA) and microdissected under a stereomicroscope (SZH; Olympus Corp., Overland Park, KA) at magnifications up to 100 , using thin steel needles and sharpened forceps (No. 5, Dumont; Fine Science Tools, Inc., Belmont, CA).

From each rabbit, a single superficial afferent arteriole was microdissected as follows. An intralobular artery was localized and gently 
pulled out of the slice together with the adhering tubular element. Tubular fragments were gently stripped off, taking care to avoid distortion of the arteriole and glomerulus or touching them with the forceps or needle. Once an appropriate arteriole was obtained, it was severed from the interlobular artery by cutting it with a mini-blade (George Tieman \& Co., Plainview, NY). We found that it is important to make the cut only once and precisely at a right angle to the afferent arteriole $\left(90^{\circ}\right)$ if subsequent cannulation is to be successful. Using a micropipette, the arteriole was transferred to a temperature-regulated chamber mounted on the stage of an inverted microscope (IMT-2; Olympus Corp.).

The method of cannulation of the afferent arteriole was described previously $(16,17)$. Fig. 1 shows a microperfused afferent arteriole with its glomerulus intact. The afferent arteriole was drawn into the holding pipette that had a constriction (internal diameter, $14 \mu \mathrm{m}$ ). The tip of the perfusion pipette (internal diameter, $8 \mu \mathrm{m}$ ) was advanced into the lumen and perfusion was begun. Then, the pressure pipette (outer diameter at the tip, $\sim 2-3 \mu \mathrm{m}$ ), which was filled with $\mathrm{NaCl}$ solution containing 5\% FD\&C green and $4 \% \mathrm{KCl}(\mathrm{pH} 7 ; 300 \mathrm{mosmol} / \mathrm{kg})$, was advanced into the arteriole through the opening of the perfusion pipette. The pressure pipette was observed under the microscope during application of various internal pressures. The pressure at which neither the colored solution flowed into the arteriole nor the intraluminal fluid into the pressure pipette was taken as being equal to the pressure in the afferent arteriole. This method, known as Landis technique, was used because it has been reported that pressure measurement by a servo-nul system is not accurate with this arrangement of pipettes (17). To facilitate exchange of the arteriolar perfusate, an exchange pipette was laid between the pressure and perfusion pipettes so that its tip was positioned near that of the perfusion pipette. Through this exchange pipette, the perfusion solution could readily be introduced into the perfusion pipette, and, if desired, the composition of the perfusate could easily be changed. The perfusate was oxygenated medium $199-5 \%$ BSA, and perfusion pressure was maintained at $60 \mathrm{mmHg}$ throughout the experiment. The driving force of the perfusate was provided by a pressurized tank of $95 \% \mathrm{O}_{2}$ and $5 \% \mathrm{CO}_{2}$ attached to the proximal end of the perfusion pipette. An air regulator positioned between the tank and the perfusion pipette was manipulated to control the intraluminal pressure at $60 \mathrm{mmHg}$ as measured with the pressure pipette.
The bath was identical to the arteriolar perfusate and exchanged continuously. Microdissection and cannulation of the afferent arteriole were completed within $90 \mathrm{~min}$ at $8^{\circ} \mathrm{C}$, after which the temperature of the bath was gradually raised to $37^{\circ} \mathrm{C}$ for the rest of the experiment. Once it had stabilized, a 30-min equilibration period was allowed before taking any measurements. When the temperature reached about $36^{\circ} \mathrm{C}$, the arteriole exhibited spontaneous cyclic contractions which disappeared during the equilibration period, allowing us to measure the stable luminal diameter. Images of the afferent arteriole were displayed at magnifications up to 1,980 and recorded with a video system consisting of a camera adaptor with a $3.3 \times$ photo-eyepiece, black and white charge-coupled device camera (NC 70; Dage-MTI, Inc., Michigan, IN), monitor (BWM 112; Javelin Electronics Inc., Torrance, CA), and video recorder (EDV-9500; Sony). The diameter at the most constricted point was measured with an image-analysis system (Fryer, Carpentersville, IL).

\section{Experimental protocol}

1. Response to AII. After the 30-min equilibration period, continuous bath exchange was stopped, and the entire bath was gently aspirated and replaced with $1 \mathrm{ml}$ of warm medium containing AII (Sigma Chemical Co.) at $10^{-8}, 10^{-7}$, or $10^{-6} \mathrm{M}$. Bath exchange was immediately resumed using medium containing the same concentration of AII and the arteriole observed for $3 \mathrm{~min}$. The arteriole was washed with plain medium 199-5\%BSA for $10 \mathrm{~min}$, after which the same concentration of AII was repeated. The diameter was measured before each application of AII and $5 \mathrm{~s}, 1$, and $3 \mathrm{~min}$ afterward. Because of the possibility of tachyphylaxis, we tested only a single concentration with a given arteriole.

2. Pretreatment with saralasin. To assure specificity of the response to AII, we examined the effect of saralasin (Sigma Chemica Co.), an antagonist of AII, on AII-induced vasoconstriction. After the equilibration period, the bath was exchanged continuously with medium containing $10^{-5} \mathrm{M}$ saralasin. $3 \mathrm{~min}$ later, the effect of AII $\left(10^{-7}\right.$ M) was tested in the presence of saralasin.

3. Pretreatment with cyclooxygenase inhibitor. Indomethacin (Sigma Chemical Co.), a cyclooxygenase inhibitor, was dissolved in saline containing $1.15 \mathrm{mg} / \mathrm{ml}$ of sodium carbonate at a final concentra-

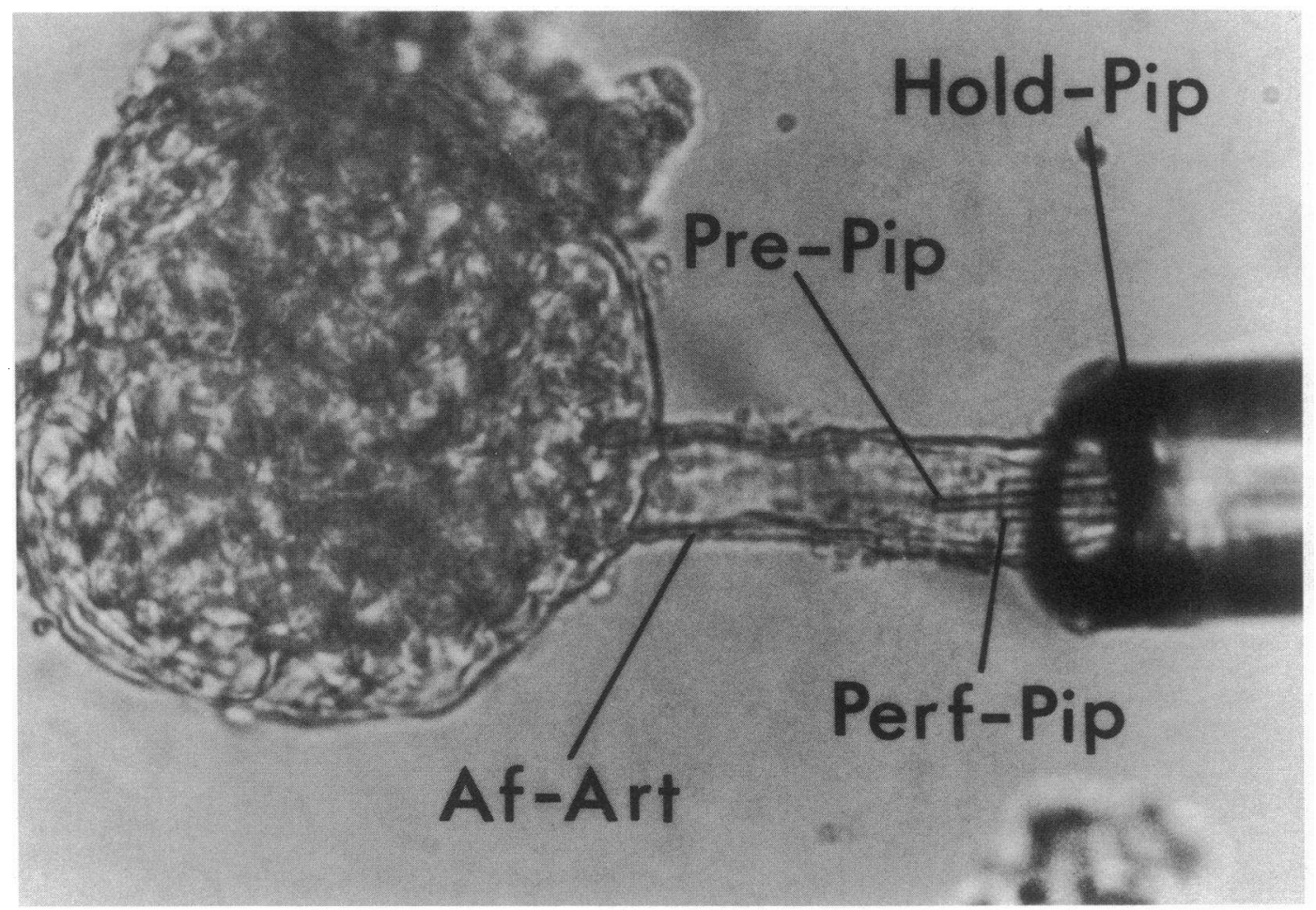

Figure 1. A Photograph of a microperfused afferent arteriole with its glomerulus intact. Hold-Pip, holding pipette; Perf-Pip, perfusion pipette; Pre-Pip pressure pipette; $A f-A r t$, afferent arteriole. Note that the distal end of the arteriole can be observed even though it is overlapped by the glomerulus. Perfusion pressure was measured with a pressure pipette (outer diameter $\sim 2-3 \mu \mathrm{m})$. 
tion of $3.57 \mathrm{mg} / \mathrm{ml}$. The rabbit received an intravenous injection of indomethacin $(5 \mathrm{mg} / \mathrm{kg}) 1 \mathrm{~h}$ before the kidney was removed. In addition, indomethacin was added to the bath and arteriolar perfusate at a final concentration of $5 \mu \mathrm{g} / \mathrm{ml}$ from the equilibration period to the end of the experiment. The effect of AII $\left(10^{-7} \mathrm{M}\right)$ was examined as described in protocol 1 . This dose of indomethacin has been reported to block the effect of arachidonic acid $\left(10^{-4} \mathrm{M}\right)$ in rabbit afferent arterioles (15).

4. Inhibition of EDRF synthesis. After the equilibration period, $N^{\text {w }}$-nitro-L-arginine ( $N$-Arg; Sigma Chemical Co.), a compound that inhibits synthesis of nitric oxide (an EDRF) $(18,19)$, was added to the bath and arteriolar perfusate at $10^{-4} \mathrm{M} .15 \mathrm{~min}$ later, $10^{-7} \mathrm{M}$ AII was tested three times in the presence of $N$-Arg. At the end of the experiments, we confirmed that acetylcholine $(10 \mu \mathrm{M})$ did not dilate arterioles preconstricted with norepinephrine $\left(5 \times 10^{-7} \mathrm{M}\right)$; the diameter decreased with norepinephrine to $38 \pm 6.2 \%$ of the control level and remained unchanged after the addition of acetylcholine $(32 \pm 6.5 \% ; n$ $=14, P>0.5$ ). We also confirmed that in nonpretreated arterioles, norepinephrine decreased luminal diameter to $44 \pm 9.6 \%$ of the control level $(n=6)$, which was reversed to $95 \pm 2.7 \%$ by acetylcholine.

Since $N$-Arg not only rendered AII-induced constrietion persistent but also reduced the basal arteriolar diameter (see Results), we tested whether $N$-Arg affected the action of AII by increasing the basal tone of the arteriole. The experimental design was the same as above except that $10^{-7} \mathrm{M}$ norepinephrine (Sigma Chemical Co.) was used instead of $N$-Arg.

5. Kidney homogenate. The arteriole was twice exposed to AII at $10^{-7} \mathrm{M}$ as described in protocol 1 . The same arteriole was then washed continuously with medium containing either kidney homogenate or $1 \%$ saline (vehicle of the kidney homogenate) for $10 \mathrm{~min}$, after which $10^{-7} \mathrm{M}$ AII was tested for the third time. To prepare the kidney homogenate, after flushing the kidney free of blood, $5 \mathrm{~g}$ of the renal cortex was excised and homogenized in $10 \mathrm{ml}$ of saline. The homogenate was centrifuged at $4^{\circ} \mathrm{C}(3,000 \mathrm{~g})$ and the supernatant diluted $100 \times$ with medium 199-5\% BSA before use.

6. Kidney homogenate combined with inhibition of EDRF synthesis. The experimental design was identical to protocol 5 except that the arteriole was pretreated with $N$-Arg $\left(10^{-4} \mathrm{M}\right)$ as described in protocol 4 .

\section{Statistics}

Values were expressed as mean $\pm \mathrm{SEM}$, and all statistical analyses were done using absolute values. A paired $t$ test was used to examine whether the diameter at a given time was different from the control value (time $0)$ or whether the change in diameter at a given time was different between applications. A two-sample $t$ test was used to examine whether the change in diameter at a given time was different between groups. Repeated measures analysis of variance was used to test overall differences between groups. $P<0.05$ was considered significant.

\section{Results}

1. Response to AII. Luminal diameter was $16.4 \pm 0.7 \mu \mathrm{m}$ ( $n$ $=23$ ) before the first application of AII and $16.6 \pm 0.7 \mu \mathrm{m}$ before the second application, showing that the basal diameter was stable. Fig. 2 shows an example of arteriolar response to AII at $10^{-6} \mathrm{M}$ and for comparison, norepinephrine-induced vasoconstriction. At $5 \mathrm{~s}$ after AII, the afferent arteriole was constricted in three segments, with the strongest being closest to the glomerulus. However, constriction soon waned, with the diameter returning to control levels by $1 \mathrm{~min}$ despite the presence of AII in the bath. In contrast, norepinephrine at $10^{-6} \mathrm{M}$ caused constriction of the entire afferent arteriole which persisted for $3 \mathrm{~min}$.

Of 23 afferent arterioles studied for $10^{-8}, 10^{-7}$, and $10^{-6} \mathrm{M}$ AII, in 6 AII-induced constriction was seen almost exclusively within $50 \mu \mathrm{m}$ from the vascular pole of the glomerulus, in 11 there were two or three segmental constrictions, with the strongest being closest to the glomerulus, and in the remaining 6 constriction was uniform along the arteriole. It seems that the site or pattern of the constriction was not different according to the concentration of AII.

Fig. 3 summarizes arteriolar responses to AII. When $10^{-8}$ M AII was first applied, luminal diameter decreased by $31 \pm 7.1 \%(n=8 ; P<0.005)$. The constriction tended to wane,
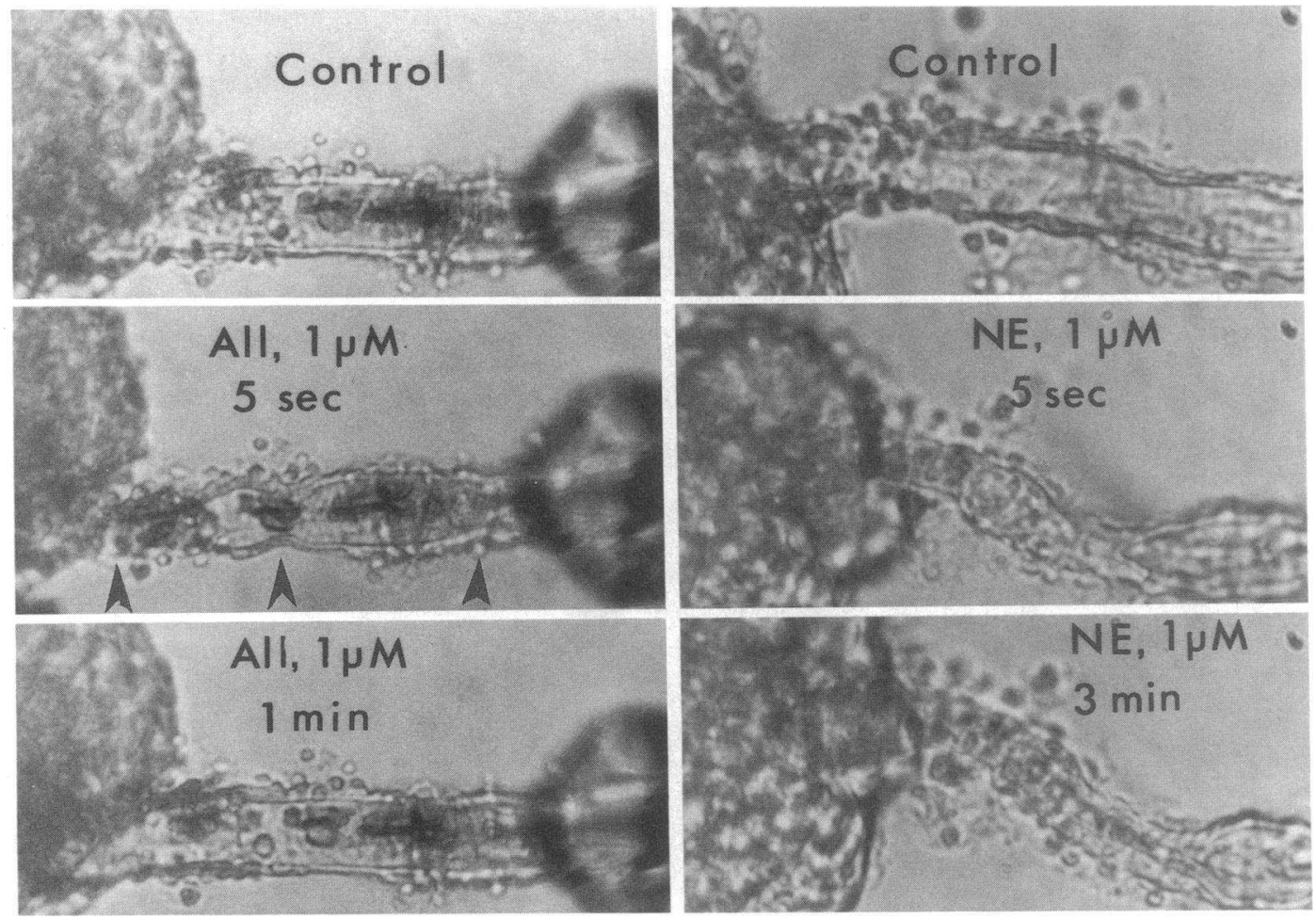

Figure 2. Example of arteriolar response to angiotensin II (left) and norepinephrine (right). Note that $1 \mu \mathrm{M}$ AII caused segmental vasoconstrictions (arrowheads; the strongest in the distal segment), which disappeared within 1 min despite the continuous presence of AII in the bath. In contrast, 1 $\mu \mathrm{M}$ norepinephrine $(N E)$ caused constriction along the entire arteriole, which persisted for $3 \mathrm{~min}$. 


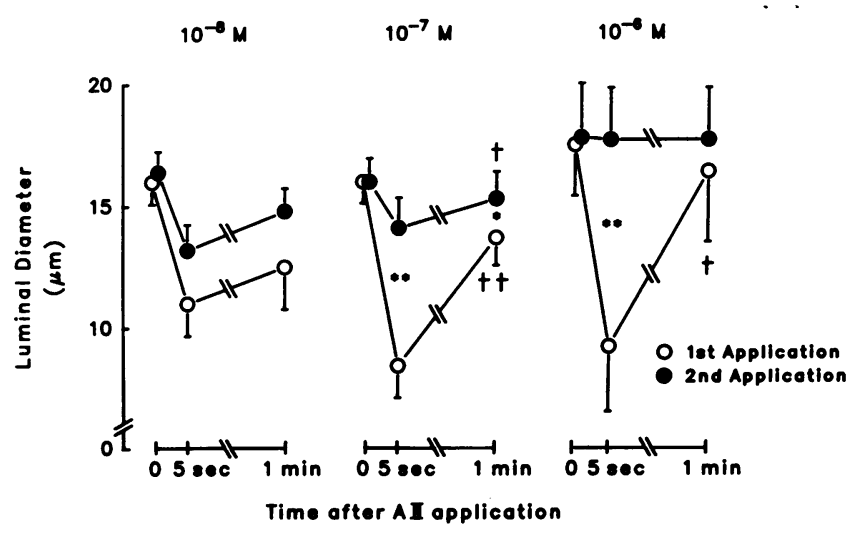

Figure 3. Effect of AII on the luminal diameter of the afferent arteriole. AII at $10^{-8}(n=8), 10^{-7}(n=9)$, or $10^{-6} \mathrm{M}(n=6)$ was applied twice with a 10-min washout period in between. Note that with $10^{-7}$ or $10^{-6} \mathrm{M}$ AII, constriction waned, and it was markedly attenuated with the second application.,$+++=P<0.05$ and 0.002 , respectively, compared with $5 \mathrm{~s} ;{ }^{*},{ }^{* *}=P<0.05$ and 0.01 , respectively, for the first versus second application.

and the second application caused a weaker constriction; however, these differences were not statistically significant. At the concentration of $10^{-7}$ or $10^{-6} \mathrm{M}$, the first application decreased luminal diameter by $49 \pm 7.0 \%(n=9 ; P<0.0001)$ or $50 \pm 10.9 \%$ $(n=6 ; P<0.01)$, respectively. The constriction waned significantly, with the diameter returning toward control levels within $1 \mathrm{~min}$, and the second application caused a significantly lesser effect compared with the first application. The higher the concentration employed, the more evident became both the transient nature of the constriction and the tachyphylaxis.

2. Saralasin pretreatment. Saralasin at $10^{-5} \mathrm{M}$ did not affect basal luminal diameter (15.2 \pm 1.5 and $15.7 \pm 1.6 \mu \mathrm{m}$ before and $3 \mathrm{~min}$ after saralasin, respectively; $n=4$.) (In some arterioles, saralasin caused a slight constriction $(\sim 15 \%)$ due to agonistic action; however, this disappeared within $1 \mathrm{~min}$ ). However, it completely blocked the vasoconstrictive action of $10^{-7}$ $\mathrm{M}$ AII; the decrease in diameter was $0.6 \pm 1.3 \%(P$ $>0.1$.

3. Pretreatment with cyclooxygenase inhibitor. Fig. 4 depicts AlI-induced changes in luminal diameter in the indomethacin-treated and nontreated groups. Pretreatment with indomethacin did not alter basal diameter $(18.2 \pm 1.1 \mu \mathrm{m} ; n$ $=6$ ), nor did it alter either the degree of vasoconstriction $(53 \pm 7.5 \%)$ or its transient nature for the first application. For the second application, AII-induced constriction was significantly attenuated as compared with the first application in the indomethacin-pretreated group as well.

4. Inhibition of EDRF synthesis. Fig. 5 shows an example of arteriolar response to pretreatment with $N$-Arg and the effect of AII after the pretreatment. With $N$-Arg pretreatment, basal luminal diameter decreased significantly by $18 \pm 3.0 \%$, falling from $19.0 \pm 1.3$ to $15.7 \pm 1.4 \mu \mathrm{m}(n=14, P<0.0001)$. ( $N$-Arginduced changes in basal luminal diameter in protocols 4 and 6 were analyzed collectively.) Furthermore, AII-induced constriction became persistent after the pretreatment with $N$-Arg.

Fig. 6 depicts the time course of the response to the first application of AII in nontreated, $N$-Arg-treated, and norepinephrine-treated arterioles. (Data from protocols 1 and 5 were

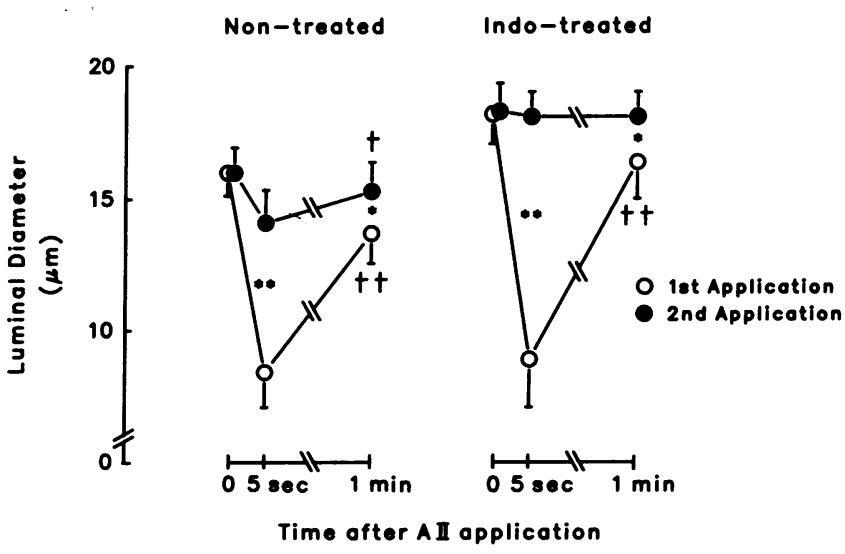

Figure 4. Effect of AII on luminal diameter in indomethacin (indo)treated $(n=6)$ and nontreated $(n=9)$ afferent arterioles. Indometha$\operatorname{cin}(5 \mu \mathrm{M})$ altered neither the transient nature of the constriction induced by AII $\left(10^{-7} \mathrm{M}\right)$ nor the tachyphylaxis.,$+++=P<0.05$ and 0.002 , respectively, compared with $5 \mathrm{~s},{ }^{*},{ }^{* *}=P<0.05$ and 0.01 , respectively, for the first versus second application. There were no significant differences between the two groups $(P>0.1)$.

combined for the nontreated group, as were data from protocols 4 and 6 for the $N$-Arg-treated group, since the experimental design was the same as for the first application.) In the nontreated group, luminal diameter decreased by $54 \pm 5.2 \%$ at $5 \mathrm{~s}$ after AII application ( $P<0.0001 ; n=20)$; however, constriction then waned, with the decrease in diameter from time 0 becoming $20 \pm 3.4$ and $11 \pm 1.9 \%$ at 1 and 3 min, respectively. In the $N$-Arg-treated group, the diameter decreased by $86 \pm 5.8 \%$ at $5 \mathrm{~s}(n=14 ; P<0.0001)$. The arterioles stayed constricted thereafter, with the decrease in diameter from time 0 being $82 \pm 6.9$ and $72 \pm 6.7 \%$ at 1 and $3 \mathrm{~min}$, respectively. At each time point, the decrease in diameter $(\Delta)$ was significantly larger in the $N$-Arg-treated group. Pretreatment with norepinephrine reduced the basal diameter by $17 \pm 6.7 \%$, from $18.2 \pm 1.0$ to $14.9 \pm 1.0 \mu \mathrm{m}(n=6)$, which was not different from that induced by $N$-Arg; similarly, it tended to augment AII-induced constriction at $5 \mathrm{~s}(89 \pm 8.2 \%)$. However, vasoconstriction waned thereafter, with the decrease in diameter from time 0 becoming $51 \pm 6.5$ and $27 \pm 7.0 \%$ at 1 and $3 \mathrm{~min}$, respectively. The decrease in diameter $(\Delta)$ at 1 and $3 \mathrm{~min}$ was significantly larger in the $N$-Arg-treated than in the norepinephrine-treated group.

5. Kidney homogenate. In the time controls $(n=5)$, the decrease in diameter $(\Delta)$ with the first, second, and third application was $12.2 \pm 3.8,6.4 \pm 2.5$, and $2.8 \pm 0.7 \mu \mathrm{m}$, respectively, demonstrating progression of tachyphylaxis (Fig. 7). In the experimental group $(n=6)$, when the arteriole was washed with plain medium 199-5\%BSA, the vasoconstriction induced by the second application was markedly attenuated as in the time controls. However, when the same arteriole was washed with kidney homogenate, the decrease induced by the third application was significantly larger than that induced by the second application $(6.3 \pm 1.3$ vs. $2.5 \pm 0.8 \mu \mathrm{m} ; P<0.05)$. The decrease in diameter induced by the third application was significantly greater in arterioles washed with kidney homogenate than in those washed with plain medium 199-5\%BSA $(P<0.05)$. Even after washing with kidney homogenate, however, AII-induced constriction disappeared within $1 \mathrm{~min}(\Delta)(0.8 \pm 0.8 \mu \mathrm{m})$. 

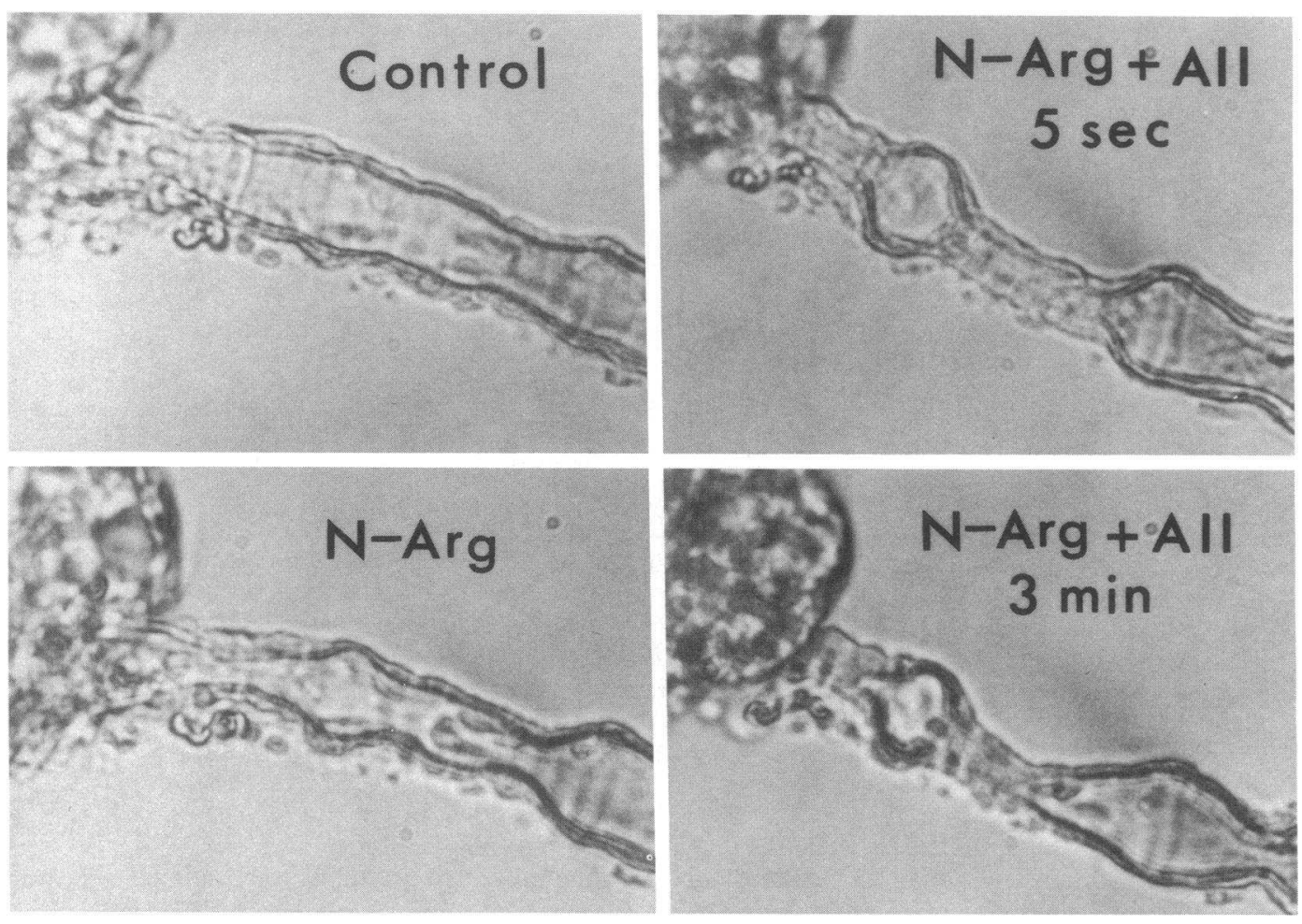

Figure 5. An example of changes in basal diameter and AII-induced constriction after pretreatment with $N$-Arg, a compound that inhibits synthesis of nitric oxide (an EDRF). Note that $N$-Arg $\left(10^{-4} \mathrm{M}\right)$ reduced basal diameter and rendered constriction induced by $\mathrm{AII}\left(10^{-7} \mathrm{M}\right)$ persistent for at least 3 min (compare with Fig. 1).

6. Kidney homogenate combined with inhibition of EDRF synthesis. Although AII-induced vasoconstriction became more persistent in the $\mathrm{N}$-Arg-treated group, repeated applications of AII caused weaker and weaker constriction in the control group (Fig. 8); the decreases $(\Delta)$ induced by the first, second, and third application were $11.8 \pm 1.4,5.4 \pm 1.0$, and

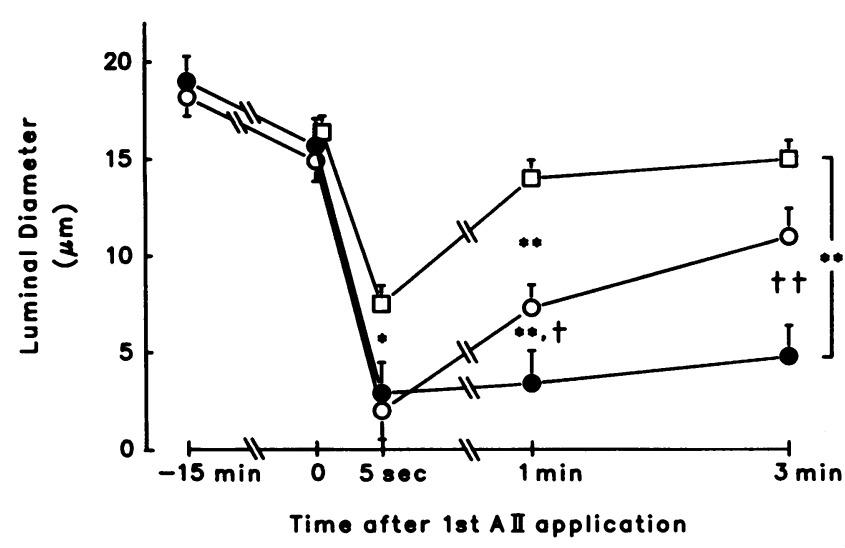

Figure 6. Time course of the response to the first application of AII in the nontreated ( $\square$ ) $(n=20)$ afferent arteriole and the arteriole pretreated with either $N$-Arg $(\bullet)(n=14)$ or norepinephrine $(N E)(0)(n$ $=6$ ). $N$-Arg at $10^{-4} \mathrm{M}$ or NE at $10^{-7} \mathrm{M}$ was added to both the bath and arteriolar perfusate $15 \mathrm{~min}$ before application of AII $\left(10^{-7} \mathrm{M}\right)$. Note that AII-induced constriction was augmented and became persistent after pretreatment with $\mathrm{N}$-Arg, whereas it still waned even after pretreatment with norepinephrine $\left(10^{-7} \mathrm{M}\right)$ despite the similar reduction in basal diameter. ${ }^{*},{ }^{* *}=P<0.05$ and 0.0001 , respectively, compared with the nontreated group;,$+++=P<0.05$ and 0.002 , respectively, compared with the NE-treated group.
$4.1 \pm 0.7 \mu \mathrm{m}$, respectively. In the experimental group, when the arteriole was washed with plain medium 199-5\%BSA, the constriction induced by the second application was significantly attenuated as in the controls. However, when the same arteriole was washed with kidney homogenate, the third application caused a significantly greater response than the second application $(9.2 \pm 1.5$ vs. $7.0 \pm 1.4 \mu \mathrm{m} ; P<0.05)$. The decrease induced by the third application was significantly larger in arterioles washed with kidney homogenate than in those washed with plain medium $199-5 \%$ BSA $(P<0.02)$.

\section{Discussion}

Our observations clearly demonstrate that $N$-Arg, a compound that inhibits nitric oxide (an EDRF), decreases the luminal

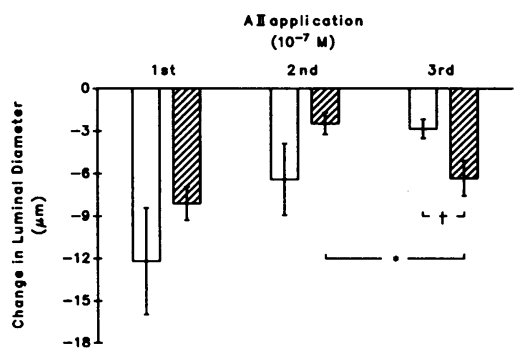

Figure 7. Changes in luminal diameter by repeated applications of AII and the effect of washing with kidney homogenate. Repeated applications of AII caused weaker and weaker constriction when the arteriole was washed with plain me-

dium 199-5\% BSA between applications (Control) $(\square)(n=5)$. When the arteriole was washed with kidney homogenate between the second and third applications $(\square)(n=6)$, AII tachyphylaxis was reversed. *, $P<0.05$ vs. the second application;,$+ P<0.05$ vs. control group. 


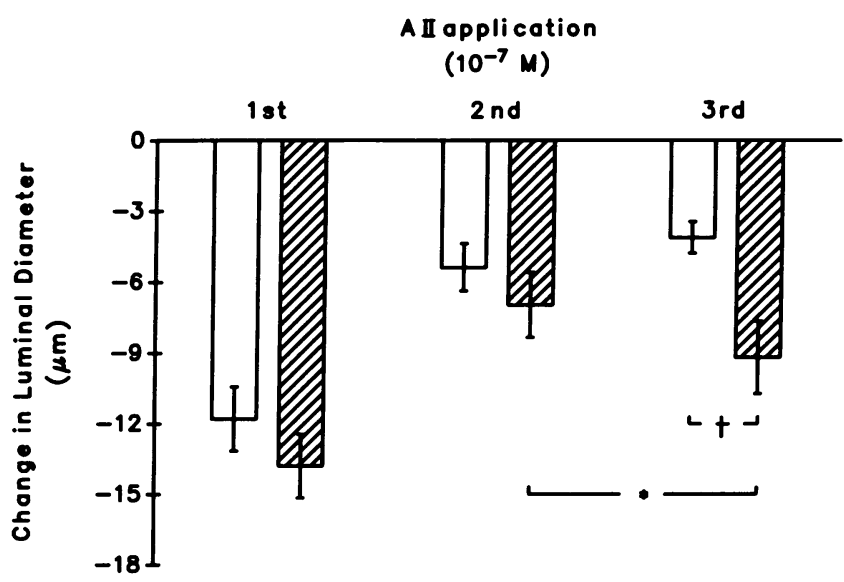

Figure 8 . Changes in luminal diameter by repeated applications of AII and the effect of washing with kidney homogenate in arterioles pretreated with $N$-Arg. Even after pretreatment with $N$-Arg, repeated applications of AII caused a weaker and weaker response when the arteriole was washed with plain medium 199-5\%BSA (Control) (ㅁ) (n $=7$ ). When the arteriole was washed with kidney homogenate between the second and third applications ( ()$\left.^{2}\right)(n=7)$, progression of AII tachyphylaxis was prevented. ${ }^{*}, P<0.05$ vs. the second application;,$+ P<0.02$ vs. the control group.

diameter of the microperfused rabbit afferent arteriole and augments the vasoconstrictive action of AII. Thus, this study provides evidence that the afferent arteriole, a small resistance vessel ( $\sim 20 \mu \mathrm{m}$ in diameter), produces EDRF which in turn plays an important role in the control of vascular tone where it is produced.

It has been shown consistently that AII constricts the afferent arteriole in situ in preparations of rat juxtamedullary nephrons (6) and split hydronephrotic kidneys (7). In contrast, using an isolated rabbit afferent arteriole with the proximal end cannulated and the distal segment (close to the glomerulus) occluded, Edwards (20) reported that increasing doses of AII $\left(10^{-12}-10^{-6} \mathrm{M}\right)$ had no effect on luminal diameter. However, when a similar technique was applied to the rat, AII caused a dose-dependent decrease in the afferent arteriolar luminal diameter (8), suggesting that species differences may be involved. In this study, we observed clear vasoconstriction when AII was first applied. However, this constriction was localized mainly (though not totally) to the distal segment close to the glomerulus, which may be why Edwards did not observe such a response. It may also be that since AII induces strong tachyphylaxis, testing the effect with increasing doses did not reveal a proper dose-response relationship.

In contrast to the constriction of the entire afferent arteriole induced by norepinephrine, AII-induced constriction was strongest in the segment close to the glomerulus for reasons that are not clear. Segmental constriction by AII has also been observed in the isolated rat afferent arteriole (8) as well as in vivo (21). In rat juxtamedullary nephrons perfused in vitro, however, the decrease in diameter was rather uniform along the afferent arteriole (6). The reason for this discrepancy is not clear, but may be related to the dose employed, the nephron population studied (superficial versus juxtamedullary afferent arteriole) and/or the preparation employed (in situ versus ex situ).
The most striking observation of this study is that pretreatment with $\mathrm{N}$-Arg not only reduced basal diameter but also rendered AII-induced vasoconstriction persistent. In addition, it augmented the vasoconstrictive action of AII at $5 \mathrm{~s}$. Since $\mathrm{N}$-Arg reduced basal diameter, its effect on AII-induced constriction could be due to the increased basal tone of the arterioles. It has been reported that pretreatment with norepinephrine, serotonin, or high potassium (20-30 mM) enhanced AIIinduced constriction and prevented tachyphylaxis in other vascular beds (22), suggesting that basal tone is an important determinant of the vascular response. In this study, pretreatment with norepinephrine, which decreased basal diameter to the same extent as $\mathrm{N}$-Arg, augmented AII-induced constriction. However, even after pretreatment with norepinephrine, AlI-induced vasoconstriction still waned, showing a significantly different time course from the $N$-Arg-treated group. Therefore, sustained arteriolar constriction by AII after $N$-Arg pretreatment may not be attributable solely to the increased basal tone of the arteriole, but rather to inhibition of EDRF synthesis.

It has recently been reported that intravenous administration of $N^{\mathrm{w}}$-monomethyl-L-arginine (L-NMMA), another compound that competitively inhibits synthesis of nitric oxide (an EDRF), increased systemic as well as renal vascular resistance (23-25). However, these in vivo studies cannot exclude the possibility that the observed changes in hemodynamics were merely secondary. For instance, since EDRF inhibits renin release (26), blocking its synthesis would increase renin release, which in turn would increase systemic vascular resistance through increased formation of AII. In our preparation, on the other hand, there are no such systemic hemodynamic or hormonal influences. Therefore, it may be reasonable to attribute the $18 \%$ decrease in basal diameter to inhibition of local synthesis of EDRF in the afferent arteriole. Thus, our results strongly suggest that the afferent arteriole produces EDRF which in turn may act locally to influence vascular tone.

Kon et al. (27) recently reported that when either acetylcholine or histamine, an EDRF-dependent vasodilator, was infused into the proximal portion of the main renal artery, renal blood flow and glomerular filtration rate increased, whereas infusion into the distal portion had either the opposite effect or no effect. They also showed that intravenous infusion of acetylcholine, which normally increases renal blood flow and glomerular filtration rate, caused no effect or a decrease when the main renal artery was denuded. These observations may indicate that the renal vasculature downstream from the main renal artery produces little EDRF in response to acetylcholine or histamine. In contrast, our results suggest that renal microvessels produce EDRF and that this EDRF is an important determinant of both the basal tone of the afferent arteriole and the vascular response to AII. In addition, Edwards (28) also observed that acetylcholine dilated afferent arterioles which were isolated and preconstricted by norepinephrine.

Although $N$-Arg augmented the vasoconstrictor action of AII and rendered it persistent, repeated applications of AII caused a weaker and weaker response (tachyphylaxis). It has been shown that AII induces tachyphylaxis in vascular strips even after functional removal of the endothelium $(12,13)$. These observations suggest that some factor(s) other than EDRF may be involved in AII tachyphylaxis. It has been reported that indomethacin reduced the degree of tachyphylaxis 
in vascular strips $(11,12)$, suggesting the involvement of prostaglandin biosynthesis. On the other hand, it has been proposed that AII tachyphylaxis is due to long lasting occupancy of the receptors. Showing reversal of tachyphylaxis with a plasma fraction rich in angiotensinase, Khairallah et al. (10) speculated that receptor-bound AII is degraded by angiotensinase, leading to conformational changes of the receptor-agonist complex which in turn may facilitate dissociation and thereby increase the number of receptors available for binding. Consistent with this hypothesis is their observation that the degree of tachyphylaxis was inversely related to angiotensinase activity in vascular strips from various species (10). In this study, indomethacin altered neither the transient nature of AII-induced vasoconstriction nor the degree of tachyphylaxis, suggesting that prostaglandin biosynthesis does not modulate the action of AII in the rabbit afferent arteriole. On the other hand, washing the arteriole with kidney homogenate reversed the tachyphylaxis (or prevented further progression). This may be compatible with the hypothesis that AII tachyphylaxis is due to long lasting receptor occupancy; however, we cannot exclude the possibility that vasoactive substances present in the kidney homogenate are also involved in the reversal of tachyphylaxis.

In order to test whether the transient constriction and tachyphylaxis induced by AII are unique to the rabbit, we also microperfused rat afferent arterioles using the same method. We found that the constriction induced by $10^{-7} \mathrm{M}$ AII did wane and tachyphylaxis developed as well, although it took longer to wane (2-3 min) and the degree of tachyphylaxis was less than in the rabbit arterioles (unpublished observation). This is in contrast to the observation of Yuan et al. (8), who reported that the action of AII persisted for at least $3 \mathrm{~min}$ in rat arterioles with the proximal end cannulated and the distal end occluded. This discrepancy may be related to the fact that there is flow through the arteriole in our preparation but none in Yuan's. Since the shear stress associated with flow may be a physiological stimulus of EDRF synthesis and release $(29,30)$, the action of AII may be different between the two preparations.

This study supports EDRF as a local regulator of afferent arteriolar resistance. The afferent arteriole is not only a small resistance vessel but also a site of both myogenic response (31, 32 ) and tubuloglomerular feedback $(16,32)$, two intrinsic mechanisms of renal autoregulation. In addition, it releases renin, which cleaves angiotensin I from angiotensinogen, the first step in activation of the renin-angiotensin system. Thus, local production of EDRF in the afferent arteriole may play an important role in the regulation of renal hemodynamics as well as systemic circulation, either directly by affecting afferent arteriolar tone or indirectly by inhibiting renin release (26). It is well known that when renal perfusion pressure is increased above the autoregulatory range, renal vascular resistance increases, so that renal blood flow and glomerular filtration rate remain constant. Such constriction of the renal vasculature (which is probably strongest in the afferent arteriole), combined with the same rate of flow, may increase shear stress on the endothelium, resulting in enhanced production (and release) of EDRF which in turn may participate in fine tuning of renal vascular resistance. Indeed, a preliminary report by Salom et al. (33) has shown that inhibition of EDRF synthesis by L-NMMA significantly decreased renal blood flow and glomerular filtration rate when renal perfusion pressure was kept high
(155 $\mathrm{mmHg}$ ) but not lower $(110 \mathrm{mmHg})$, suggesting that EDRF is an important regulator of renal function at high perfusion pressures. It is conceivable that a major site of action as well as production of the EDRF responsible for such changes is the afferent arteriole. Along the same lines, it may be that increased local production of EDRF in the afferent arteriole mediates or modulates inhibition of renin release induced by high renal perfusion pressures. However, further experiments are necessary in order to clarify the role of EDRF in renal autoregulation (the myogenic response and the tubuloglomerular feedback mechanism) as well as in pressure- or baroreceptor-mediated renin release.

In conclusion, we have shown that AII constricts the rabbit afferent arteriole primarily in the segment close to the glomerulus. However, the constriction is only transient, and tachyphylaxis develops rapidly. We present evidence that the transient action may be due to EDRF synthesis, while the tachyphylaxis may be the result of long lasting receptor occupancy by AII. Our results strongly suggest that locally produced EDRF may be an important regulator of vascular tone in the afferent arteriole, a small resistance vessel.

\section{Acknowledgments}

This study was supported by National Institutes of Health grant HL28982. We gratefully acknowledge the excellent assistance of Chisa Yamaguchi.

\section{References}

1. Furchgott, R. F., and J. V. Zawadzki. 1980. The obligatory role of endothelial cells in the relaxation of arterial smooth muscle by acetylcholine. Nature (Lond.). 288:373-376.

2. Furchgott, R. F. 1983. Role of endothelium in responses of vascular smooth muscle. Circ. Res. 53:557-573.

3. Vanhoutte, P. M., G. M. Rubanyi, V. M. Miller, and D. S. Houston. 1986. Modulation of vascular smooth muscle contraction by the endothelium. Annu. Rev. Physiol. 48:307-320.

4. De Nucci, G., R. Thomas, P. D'Orleans-Juste, E. Antunes, C. Walder, T. D. Warner, and J. R. Vane. 1988. Pressor effects of circulating endothelin are limited by its removal in the pulmonary circulation and by the release of prostacyclin and endothelium-derived relaxing factor. Proc. Natl. Acad. Sci. USA. 85:9797-9800.

5. Mitchell, K. D., and L. G. Navar. 1987. Superficial nephron responses to peritubular capillary infusions of angiotensins I and II. Am. J. Physiol. 252:F818F824.

6. Carmines, P. K., T. K. Morrison, and L. G. Navar. 1986. Angiotensin II effects on microvascular diameters of in vitro blood-perfused juxtamedullary nephrons. Am. J. Physiol. 251:F610-F618.

7. Steinhausen, M., R. B. Sterzel, J. T. Fleming, R. Kühn, and S. Weis. 1987. Acute and chronic effects of angiotensin II on the vessels of the split hydronephrotic kidney. Kidney Int. 31(Suppl. 20):S64-S73.

8. Yuan, B. H., J. B. Robinette, and J. D. Conger. 1990. Effect of angiotensin II and norepinephrine on isolated rat afferent and efferent arterioles. Am. J. Physiol. 258:F741-F750.

9. Ito, S., and O. A. Carretero. 1989. Vasoconstriction and tachyphylaxis with angiotensin II (AII) in isolated microperfused rabbit afferent arteriole (AF). Circulation. 80(Suppl. II):II-101a. (Abstr.)

10. Khairallah, P. A., I. H. Page, F. M. Bumpus, and R. K. Türker. 1966. Angiotensin tachyphylaxis and its reversal. Circ. Res. 19:247-254.

11. Aiken, J. W. 1974. Effects of prostaglandin synthesis inhibitors on angiotensin tachyphylaxis in the isolated coeliac and mesenteric arteries of the rabbit. Pol. J. Pharmacol. Pharm. 26:217-227.

12. Chiba, S., and M. Tsukada. 1986. Angiotensin II-induced tachyphylactic constrictions in isolated and perfused canine mesenteric arteries. Tohoku J. Exp. Med. 150:417-426.

13. Silva, E. G., A. T. Ferreira, A. C. M. Paiva, and T. B. Paiva. 1988. Angiotensin tachyphylaxis in normal and everted rings of rabbit aorta. Eur. J. Pharmacol. 153:185-190.

14. Itoh, S., O. A. Carretero, and R. D. Murray. 1985. Renin release from isolated afferent arterioles. Kidney Int. 27:762-767. 
15. Itoh, S., and O. A. Carretero. 1985. Role of the macula densa in renin release. Hypertension. 7(Suppl. I):I49-I54.

16. Ito, S., and O. A. Carretero. 1990. An in vitro approach to the study of macula densa-mediated glomerular hemodynamics. Kidney Int. 38:1206-1210.

17. Osgood, R. W., M. Patton, M. J. Hanley, M. Venkatachalam, H. J. Reineck, and J. H. Stein. 1983. In vitro perfusion of the isolated dog glomerulus. Am J. Physiol. 244:F349-F354.

18. Moore, P. K., O. A. al-Swayeh, N. W. S. Chong, R. A. Evans, and A. Gibson. 1990. L-N ${ }^{G}$-nitro arginine (L-NOARG), a novel, L-arginine-reversible inhibitor of endothelium-dependent vasodilation in vitro. Br. J. Pharmacol. 99:408-412.

19. Ishii, K., B. Chang, and J. F. Kerwin, Jr. 1990. N omega-nitro-L-arginine: a potent inhibitor of endothelium-derived relaxing factor formation. Eur. J. Pharmacol. 176:219-223.

20. Edwards, R. M. 1983. Segmental effects of norepinephrine and angiotensin II on isolated renal microvessels. Am. J. Physiol. 244:F526-F534.

21. Wilson, S. K. 1987. A comparison of the effects of angiotensin II and norepinephrine on intrarenal arteries in the rat. Kidney Int. 31(Suppl. 20):S193S199.

22. Juul, B., C. Aalkjær, and M. J. Mulvany. 1987. Responses of femoral resistance vessels to angiotensin in vitro. Eur. J. Pharmacol. 135:61-68.

23. Rees, D. D., R. M. J. Palmer, and S. Moncada. 1989. Role of endothelium-derived nitric oxide in the regulation of blood pressure. Proc. Natl. Acad. Sci. USA. 86:3375-3378.

24. Whittle, B. J. R., J. Lopez-Belmonte, and D. D. Rees. 1989. Modulation of the vasodepressor actions of acetylcholine, bradykinin, substance $P$ and endothe- lin in the rat by a specific inhibitor of nitric oxide formation. Br. J. Pharmacol. 98:646-652.

25. Tolins, J. P., R. M. Palmer, S. Moncada, and L. Raij. 1990. Role of endothelium-derived relaxing factor in regulation of renal hemodynamic responses. Am. J. Physiol. 258:H655-H662.

26. Vidal, M. J., J. C. Romero, and P. M. Vanhoutte. 1988. Endothelium-derived relaxing factor inhibits renin release. Eur. J. Pharmacol. 149:401-402.

27. Kon, V., R. C. Harris, and I. Ichikawa. 1990. A regulatory role for large vessels in organ circulation. J. Clin. Invest. 85:1728-1733.

28. Edwards, R. M. 1985. Response of isolated renal arterioles to acetylcholine, dopamine, and bradykinin. Am. J. Physiol. 248:F183-189.

29. Gerove, M., V. Smiesko, J. Cero, and E. Barta. 1983. Dilatation of conduit coronary artery induced by high blood flow. Physiol. Bohemoslov. 32:55-63.

30. Rubanyi, G. M., J. C. Romero, and P. M. Vanhoutte. 1986. Flow-induced release of endothelium-derived relaxing factor. Am. J. Physiol. 250:H1145H1149.

31. Hayashi, K., M. Epstein, and R. Loutzenhiser. 1989. Pressure-induced vasoconstriction of renal microvessels in normotensive and hypertensive rats. Circ. Res. 65:1475-1484.

32. Casellas, D., and L. C. Moore. 1990. Autoregulation and tubuloglomerular feedback in juxtamedullary glomerular arterioles. Am. J. Physiol. 258:F660 F669.

33. Salom, M. G., V. Lahera, F. J. Fenoy, R. Roman, and J. C. Romero. 1990. Role of endothelium-derived relaxing factor (EDRF) in the renal response to changes in renal perfusion pressure. J. Am. Soc. Nephrol. 1:670a. (Abstr.) 